\title{
3D Magnetism Maps Reveal Exotic Topologies
}

\section{A recently developed $x$-ray-based technique for imaging the spin patterns inside 3D structures uncovers previously unseen patterns.}

\section{By Katherine Wright}

S ince their invention, magnetic information-storage devices have been shrinking. This size reduction has allowed technologists to squeeze more bits into smaller and smaller gadgets. Device makers would like to increase the storage density of magnetic devices further, but there is a hitch: they have almost reached the miniaturization limit of the $2 \mathrm{D}$ materials that they typically use. To solve that problem, researchers plan to move to the third dimension, a change that requires new methods to monitor and characterize

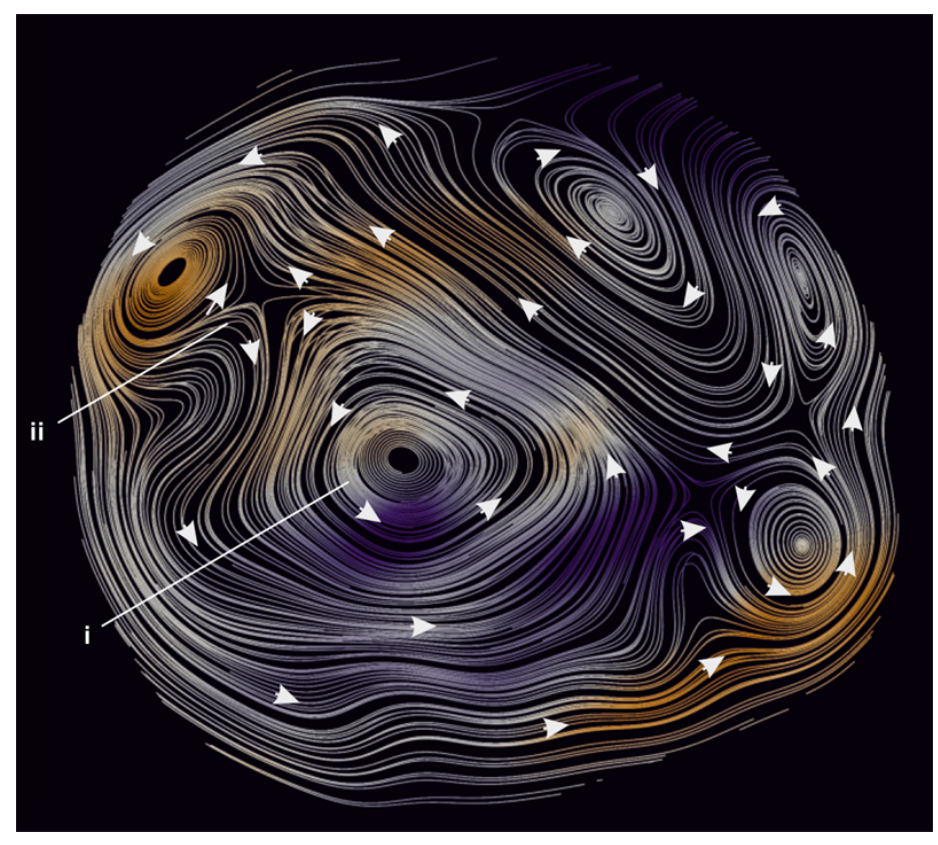

This magnetic tomography map reveals the swirls in magnetization that occur within a slice through a 5- $\mu \mathrm{m}$-diameter cylinder made of gadolinium cobalt. The long arrows point to two types of vortices. Credit: C. Donnelly et al., Nature (2017) magnetization patterns, something Claire Donnelly at the University of Cambridge, UK, and her colleagues at the Swiss Federal Institute of Technology (ETH) in Zurich and at the Paul Scherrer Institute, Switzerland, are developing. Donnelly shared her latest results-which include uncovering a new 3D magnetic pattern-at the recent CMD2020GEFES conference, an online meeting that hosted talks on all aspects of condensed matter physics.

"Most of the [imaging] work that's been done so far on magnetic materials has been carried out on planar systems or surfaces," Donnelly says. "Moving to 3D offers a huge number of opportunities for advances." To reach the third dimension, researchers could, for example, stack many hundreds of 100-nm-sized magnetic "bricks" on top of each other to create a micrometer-sized pillar.

Magnetic imaging techniques have existed for nearly a century, but they typically only probe a material's surface. For example, magnetic force microscopy captures the stray magnetic fields protruding from a material and then uses them to map the pointing direction of magnetic domains. The most widely applied of these $2 \mathrm{D}$ techniques can only probe the magnetization of a film of material to a depth of a few hundred nanometers, which is too shallow to see what is happening inside micrometer-sized 3D structures, Donnelly says. The techniques that she has helped develop provide the possibility to probe deeper inside a magnetic material.

In the first method, called magnetic tomography, a circularly polarized, short-wavelength $\mathrm{x}$-ray beam is focused on a spot on a 3D magnetic object. Circularly polarized $x$ rays are sensitive to the magnetic properties of a material because of spin-orbit 
coupling. And when these $x$ rays interact with an object, their absorption depends on how the object's spins are orientated (spin texture) and on whether the $x$ rays have left or right circular polarization. The $x$-ray beam is then scanned across the object, which is rotated and tilted, allowing the group to image a series of $2 \mathrm{D} x$-ray projections of the spin texture at different sample orientations. The projections are then plugged into an algorithm that renders the object's magnetic structure in 3D.

Using the technique, Donnelly and her colleagues have successfully mapped the 3D spin texture of a 5 - $\mu$ m-diameter cylinder made of gadolinium cobalt. In the experiment, they achieved a resolution of $100 \mathrm{~nm}$, meaning they could distinguish on this length scale changes in the pointing direction of neighboring magnetic domains within the cylinder. This feat was a first for visualizing 3D spin textures, says Donnelly. Inside the cylinder, the team spotted a complex magnetic pattern consisting of vortices and antivortices, along with so-called Bloch point singularities, which are points where the magnetization vanishes. The magnetic pattern surrounding the singularity has never been seen before, Donnelly says. They also recently discovered a new texture in this cylinder, a so-called magnetic vortex ring made up of a loop of pairs of vortices and antivortices.

Despite allowing these new findings, magnetic $x$-ray tomography has a downside-it can only be used to look at static spin textures. To probe magnetization dynamics the sample has to be excited and then imaged without interrupting this excitation. That requirement is "too difficult" for tomography, Donnelly says, because it requires taking two datasets at different sample orientations, as well as other factors. That issue spurred her and her colleagues to develop a second technique, called magnetic laminography, which they demonstrated earlier this year.

Laminography is similar to tomography, but the rotation axis of the sample is no longer perpendicular to the $\mathrm{x}$-ray beam. This change means that the sample can be angled such that all three spatial dimensions can be imaged in one shot. "Laminography gets around the need to measure two datasets and it also works better for imaging flat or extended structures, which are the sample geometries most people study," Donnelly says. The team showed that they could map the magnetization dynamics of a $5-\mu \mathrm{m}$-thick disk with a spatial resolution of $50 \mathrm{~nm}$ and a time resolution of $70 \mathrm{ps}$. Now, Donnelly says, her team can watch what's going on in 3D when the material is excited by a magnetic field.

These new 3D imaging techniques could potentially allow researchers to uncover yet more interesting spin textures, which they could use to improve magnetic storage devices. "The magnetic textures in 3D systems can be more stable than the patterns seen in planar magnetic films," Donnelly says, allowing for the materials to transport higher velocity spin waves because of reduced instabilities. Thus, this advance could lead to devices with speedier reading and writing capabilities. "That gives [researchers] the potential to do what [they] already do with these materials much faster, but there is also the potential-which is more exciting-to do completely new things."

Katherine Wright is a Senior Editor for Physics. 